\title{
Wavelet Multiscale Analysis for Hedge Funds: Scaling and Strategies
}

\author{
T. Conlon ${ }^{\mathrm{a}}$, M. Crane ${ }^{\mathrm{a}}$, H.J. Ruskin ${ }^{\mathrm{a}}$, \\ ${ }^{a}$ Dublin City University, Glasnevin, Dublin 9, Ireland
}

\begin{abstract}
The wide acceptance of Hedge Funds by Institutional Investors and Pension Funds has led to an explosive growth in assets under management. These investors are drawn to Hedge Funds due to the seemingly low correlation with traditional investments and the attractive returns. The correlations and market risk (the Beta in the Capital Asset Pricing Model) of Hedge Funds are generally calculated using monthly returns data, which may produce misleading results as Hedge Funds often hold illiquid exchange-traded securities or difficult to price over-thecounter securities. In this paper, the Maximum Overlap Discrete Wavelet Transform (MODWT) is applied to measure the scaling properties of Hedge Fund correlation and market risk with respect to the $\mathrm{S} \& \mathrm{P} 500$. It is found that the level of correlation and market risk varies greatly according to the strategy studied and the time scale examined. Finally, the effects of scaling properties on the risk profile of a portfolio made up of Hedge Funds is studied using correlation matrices calculated over different time horizons.
\end{abstract}

Key words: Scaling, Cross-Correlation, Wavelets, Hedge Funds, Econophysics PACS: 89.65.GhEconomics; econophysics, financial markets, business and management, 89.65.-sSocial and economic systems, 89.75.-kComplex systems

\section{Introduction}

A Hedge Fund is a lightly-regulated private investment vehicle that may utilise a wide range of investment strategies and instruments. These funds may use short positions, derivatives, leverage and charge incentive-based fees. Normally, they are structured as limited partnerships or offshore investment companies. Hedge Funds pursue positive returns in all markets and hence are described as "Absolute Return" strategies.

\footnotetext{
Email addresses: tconlon@computing.dcu.ie (T. Conlon), mcrane@computing.dcu.ie (M. Crane), hruskin@computing.dcu.ie (H.J. Ruskin).
} 
Hedge Funds are utilised by pension funds, high net-worth individuals and institutions, partly due to their low correlation with traditional long-only investment strategies. The incentive-based performance fees, earned by hedge fund managers, align the interest of the hedge fund manager with that of the investor. The performance of Hedge Funds has been impressive, with the various Hedge Fund indices providing higher returns, with lower volatility, than traditional assets over many years. As of the end of the fourth quarter of 2007, the total assets managed by hedge funds world-wide is estimated at $\$ 2.065$ trillion [1].

In addition to their attractive returns, many hedge funds claim to provide significant diversification benefits. Moderate correlations between Hedge Funds and traditional asset classes, using monthly returns data, have been reported [2,3]. Hence, the market exposure of Hedge Funds to traditional assets classes has also been found to be low. However, these results were based upon monthly returns data and may be misleading. Hedge Funds may hold illiquid exchange traded assets or over the counter (OTC) securities. These may be priced using the last traded price, which may not have traded at or even near the end of the month, resulting in non-synchronous pricing. Furthermore, OTC securities may not have publicly available prices but instead rely on broker prices. Due to the large performance fees charged by hedge funds, using stale or non-synchronous data may be of great benefit to the manager, at the expense of the investor.

Risk control techniques for portfolios of hedge funds have been studied by various authors. The threshold point of self-organised criticality was used as a control parameter of risk exposure [4] for a portfolio of Hedge Funds. The correlation matrix between the returns of Hedge Funds was used [5] to characterise Hedge Fund strategies using Random Matrix Theory (RMT) and a minimum spanning tree. Random Matrix Theory was applied to the correlation matrix between Hedge Funds [6] where the components of the eigenvalues deviating from RMT were shown to correspond to distinct groups of strategies that are applied by Hedge Fund managers. The information in these deviating eigenvalues was then applied to construct a portfolio with reduced levels of risk. A downside-risk ${ }^{1}$ framework was used [7] for the study of Hedge Fund risk, taking into account the inherent asymmetry in Hedge Fund returns distributions.

Multiscale analysis of the covariance between time series has been applied to the physical sciences in numerous cases (eg. [8-10]). Wavelet multiscale analysis has also been used by various authors to decompose economic and financial time series into orthogonal time-scale components of varying granularities. Wavelet decomposition was used [11] to analyse economic relationships in order to investigate anomalies that were previously unexplained. The scaling properties of foreign exchange volatility were examined [12] using wavelet multiscaling techniques where it was shown that the correlation between foreign exchange volatilities increases for scales from 20 minutes to daily. Wavelet multiscaling was applied as a filtering technique [13] to remove intraday seasonalities from data in the estimation of volatility models. The systematic risk in a Capital Asset Pricing Model was estimated over different granularities [14] where it was shown that the return of a portfolio and its beta becomes stronger as the scale increases for the S\&P 500. This technqiue was then applied to the markets of various other countries [15] where similar results were also found. The dependence of stock return cross-correlations on the sampling time

1 Downside-risk measures look at how much money an investor stands to lose during adverse market conditions 
scale is known as the Epps effect [16]. This decrease of correlations between stock returns for shorter time scales was studied [17] where it was shown that asynchronicity between stock returns was not solely responsible for the Epps effect.

In this paper we use wavelet multiscaling techniques to show that the correlation between Hedge Fund strategies and the market, calculated using monthly returns data, can be significantly different to that calculated using longer time horizons from $2-4$ months to $16-32$ months. Also, the systematic market risk of different Hedge Fund strategies is different using monthly data to that calculated over longer horizons. We then show, by calculating an efficient frontier (see Section 2.1) at each time scale, the effect of scaling on the risk profile of a portfolio of Hedge Funds.

This paper is organised as follows. In the next section, we present the main results of the Capital Asset Pricing Model and Portfolio Optimisation. Section 3 introduces the Maximum Overlap Discrete Wavelet transfer and the calculation of correlation and variance at different scales. The Hedge Fund data used is described in Section 4 and Section 5 shows the empirical results obtained. Section 6 concludes the paper and gives some directions for further research.

\section{The Capital Asset Pricing Model}

The Capital Asset Pricing Model (CAPM) was developed [18], building on earlier work [19], as a model for pricing an individual asset or a portfolio. The derivation of the standard CAPM is based upon a number of assumptions such as risk-averse investors, infinite divisibility of financial assets, absence of personal income tax, unlimited short sales, unlimited borrowing and lending at the risk free rate together with the axiom that all assets are marketable (see [20]).

The CAPM states that the expected return of a security or a portfolio equals the rate on a risk-free security plus a risk premium. The latter depends linearly on the market risk exposure (i.e. the Beta of an asset, Equation 2) and the market risk premium. Hence, the expected return on a risky asset is given by:

$$
E\left(R_{i}\right)=R_{f}+\beta_{i}\left[E\left(R_{m}\right)-R_{f}\right]
$$

where $E\left(R_{i}\right)$ is the expected return on the risky asset, $E\left(R_{m}\right)$ is the expected return of the market, $R_{f}$ denotes the risk free rate and $\beta_{i}$ is the Beta of the portfolio with respect to the market portfolio $M$ (in excess of the risk free rate return) and is given by

$$
\beta_{i}=\frac{\operatorname{Cov}\left(R_{i}, R_{m}\right)}{\operatorname{Var}\left(R_{m}\right)}
$$

Equation 1 can be rewritten in terms of the risk premium by simply subtracting the risk-free rate from both sides of the equation giving,

$$
E\left(R_{i}\right)-R_{f}=\beta_{i}\left[E\left(R_{m}\right)-R_{f}\right] .
$$

The empirical version of equation 3 is given by

$$
R_{i}-R_{f}=\alpha_{i}+\beta_{i}\left[R_{m}-R_{f}\right]+\epsilon_{i}
$$

where $\alpha_{i}$ is the expected firm-specific return and $\epsilon_{i}$ is a random error term. For a more detailed treatise of the CAPM see $[14,15,18,20,21]$. 


\subsection{Portfolio Optimisation}

The diversification of an investment into independently fluctuating assets reduces its risk (see $[18,20,21])$. However, since cross-correlations between asset prices exist, the accurate calculation of the cross-correlation matrix is vitally important. The expected return on a portfolio with $N$ assets is given by

$$
\Phi=w_{0} R_{f}+\sum_{i=1}^{N} w_{i} R_{i}
$$

where $R_{i}(t)$ is the expected return on asset $i$ at time $t, w_{0}$ is the fraction of wealth invested in the risk-free security $R_{f}, w_{i}$ is the fraction of wealth invested in asset $i$ and $\sum_{i=0}^{N} w_{i}=1$. The risk associated with holding this portfolio is then given by

$$
\Omega^{2}=\sum_{i=1}^{N} \sum_{j=1}^{N} w_{i} w_{j} C_{i j} \sigma_{i} \sigma_{j}
$$

where $\sigma_{i}$ is the volatility of $R_{i}$ and $C_{i j}$ are the elements of the cross-correlation matrix. In order to find an optimal portfolio, using the Markowitz theory of portfolio optimisation [19-21], we minimise $\Omega^{2}$ under the constraint that the return on the portfolio, $\Phi$, is some fixed value. This minimisation can be implemented by using two Lagrange multipliers and leads to a set of $N$ linear equations which can be solved for $w_{i}$. If we minimise $\Omega^{2}$ for a number of different values of $\Phi$, then we can obtain a region bounded by an upward-sloping curve, called the efficient frontier with the portfolio risk on the x-axis and the expected portfolio return on the y-axis.

\section{Wavelet Analysis}

The term Wavelet was coined by Morlet and Grossman in the 1980's, some of the earliest researchers in the field. The word "Wavelet" derives from the French Ondelette meaning "Small Wave". These "Small Waves" provide an efficient means of studying the multiresolution properties of a signal, as they can be used to decompose a signal into different time horizons. Wavelet filters have been applied to many different applications such as meterology, image analysis, signal processing and financial time series $[8-15,22-$ $24]$.

The Discrete Wavelet Transform (DWT) [25-28], in particular, allows the decomposition of a signal into components of different frequency. There are two basic wavelet functions, the father wavelet $\phi$ and the mother wavelet $\psi$. The formal definition of the father and mother wavelets are the functions:

$$
\begin{aligned}
& \phi_{j, k}(t)=2^{\frac{j}{2}} \phi\left(2^{j} t-k\right) \\
& \psi_{j, k}(t)=2^{\frac{j}{2}} \psi\left(2^{j} t-k\right)
\end{aligned}
$$

where $j=1, \ldots J$ in a $J$-level decomposition. The father wavelet integrates to 1 and reconstructs the longest time-scale component of the series, whereas the mother wavelet 
integrates to 0 and is used to describe the deviations from the trend. The wavelet representation of a discrete signal $f(t)$ in $L^{2}(R)$ is given by:

$$
f(t)=\sum_{k} s_{J, k} \phi_{J, k}(t)+\sum_{k} d_{J, k} \phi_{J, k}(t)+\ldots+\sum_{k} d_{1, k} \phi_{1, k}(t)
$$

where $J$ is the number of multiresolution levels (or scales) and $k$ ranges from 1 to the number of coefficients in the specified level. The coefficients $s_{J, k}$ and $d_{J, k}$ are the smooth and detail component coefficients respectively and are given by

$$
\begin{aligned}
s_{J, k} & =\int \phi_{J, k} f(t) d t \\
d_{j, k} & =\int \psi_{j, k} f(t) d t(j=1, \ldots J)
\end{aligned}
$$

Each of the sets of the coefficients $S_{J}, d_{J}, d_{J-1}, \ldots d_{1}$ is called a crystal.

The MODWT $[8,12,22,28]$ is a linear filter that transforms a series into coefficients related to variations over a set of scales. Like the Discrete Wavelet Transform (DWT) it produces a set of time-dependent wavelet and scaling coefficients with basis vectors associated with a location $t$ and a unitless scale $\tau_{j}=2^{j-1}$ for each decomposition level $j=1, \ldots, J_{0}$. The MODWT has, unlike the DWT, a high level of redundancy and is nonorthogonal. It retains downsampled ${ }^{2}$ values at each level of the decomposition that would be discarded by the DWT. The MODWT can also handle any sample size N, whereas the DWT restricts the sample size to a multiple of $2^{j}$.

Decomposing a signal using the MODWT to $J$ levels theoretically involves the application of $J$ pairs of filters. The filtering operation at the $j^{\text {th }}$ level consists of applying a rescaled father wavelet to yield a set of detail coefficients

$$
\tilde{D}_{j, t}=\sum_{L_{j}-1}^{l=0} \tilde{\psi}_{j, l} f_{t-l}
$$

and a rescaled mother wavelet to yield a set of scaling coefficients

$$
\tilde{S}_{j, t}=\sum_{L_{j}-1}^{l=0} \tilde{\phi}_{j, l} f_{t-l}
$$

for all times $t=\ldots,-1,0,1, \ldots$, where $f$ is the function to be decomposed [28]. The rescaled mother $\tilde{\psi}_{j, l}=\frac{\psi_{j, l}}{2^{j}}$ and father $\tilde{\phi}_{j, t}=\frac{\phi_{j, l}}{2^{j}}$ wavelets for the $j^{\text {th }}$ level are a set of scale-dependent localized differencing and averaging operators and can be regarded as rescaled versions of the originals. The $j^{\text {th }}$ level equivalent filter coefficients have a width $L_{j}=\left(2^{j}-1\right)(L-1)+1$, where $L$ is the width of the $j=1$ base filter. In practice the filters for $j>1$ are not explicitly constructed because the detail and scaling coefficients can be calculated using an algorithm that involves the $j=1$ filters operating on the $j^{\text {th }}$ level scaling coefficients to generate the $j+1$ level scaling and detail coefficients [28].

$\overline{2}$ Downsampling or decimation of the wavelet coefficients retains half of the number of coefficients that were retained at the previous scale. Downsampling is applied in the Discreate Wavelet Transform 


\subsection{Wavelet Variance}

The wavelet variance $\nu_{f}^{2}\left(\tau_{j}\right)$ is defined as the expected value of $\tilde{D}_{j, t}^{2}$ if we consider only the non-boundary coefficients. An unbiased estimator of the wavelet variance is formed by removing all the coefficients that are affected by the boundary conditions ${ }^{3}$ and is given by:

$$
\nu_{f}^{2}\left(\tau_{j}\right)=\frac{1}{M_{j}} \sum_{t=L_{j}-1}^{N-1} \tilde{D}_{j, t}^{2}
$$

where $M_{j}=N-L_{j}+1$ is the number of non-boundary coefficients at the $j^{\text {th }}$ level [28]. The wavelet variance decomposes the variance of a process on a scale by scale basis and allows us to explore how a signal behaves over different time horizons.

\subsection{Wavelet Covariance and Correlation}

The wavelet covariance between functions $f(t)$ and $g(t)$ is similarly defined to be the covariance of the wavelet coefficients at a given scale. The unbiased estimator of the wavelet covariance at the $j^{\text {th }}$ scale is given by

$$
\nu_{f g}\left(\tau_{j}\right)=\frac{1}{M_{j}} \sum_{t=L_{j}-1}^{N-1} \tilde{D}_{j, t}^{f(t)} \tilde{D}_{j, t}^{g(t)}
$$

where all the wavelet coefficients affected by the boundary are removed [28] and $M_{j}=$ $N-L_{j}+1$.

The MODWT estimate of the wavelet cross correlation between functions $f(t)$ and $g(t)$ may then be calculated using the wavelet covariance and the square root of the wavelet variance of the functions at each scale $j$. The MODWT estimator [22] of the wavelet correlation is then given by:

$$
\rho_{f g}\left(\tau_{j}\right)=\frac{\nu_{f g}\left(\tau_{j}\right)}{\nu_{f}\left(\tau_{j}\right) \nu_{g}\left(\tau_{j}\right)}
$$

In the CAPM model, the wavelet Beta estimator, at scale $j$, is defined as

$$
\beta_{f m}\left(\tau_{j}\right)=\frac{\nu_{f m}\left(\tau_{j}\right)}{\nu_{m}^{2}\left(\tau_{j}\right)}
$$

where $\nu_{f m}\left(\tau_{j}\right)$ is the covariance between an asset $f$ and the market $m$, and $\nu_{m}\left(\tau_{j}\right)$ is the variance of the return on the market portfolio at scale $j$.

\section{Data}

To enable us to study the market risk and correlations of Hedge Funds at different time scales, we use returns from the Credit Suisse/Tremont indices [29] from April 1994

3 The MODWT treats the time series as if it were periodic using "circular boundary conditions". There are $L_{j}$ wavelet and scaling coefficients that are influenced by the extension, which are refered to as the boundary coefficients. 
to October 2006, a total of 151 months. These indices use the Credit Suisse/Tremont database, which tracks over 4500 funds and consists only of funds with a minimum of USD 50 million under management, a 12 month track record and audited financial statements. The indices are calculated and rebalanced on a monthly basis and are net of all performance and management fees.

The funds in the Credit Suisse/Tremont database are separated into 10 primary categories based on their investment style. From this universe, Credit Suisse/Tremont selects a subset of funds for inclusion in the sub-indices such that each sub-index represents at least $85 \%$ of the assets under management in that respective category. The sub-indices are Convertible Arbitrage, Dedicated Short Bias, Emerging Markets, Equity Market Neutral, Event-Driven, Fixed Income Arbitrage, Global Macro, Long/Short Equity, Managed Futures and Multi-Strategy. Each of these strategies is distinct both in the instruments used and the types of markets traded and detailed descriptions of each can be found in [29-31].

The S\&P 500 index is chosen to represent the "Market". The S\&P 500 is a cap-weighted equity index consisting of large publicly held companies and trade on major US stock exchanges such as the New York Stock Exchange and NASDAQ. Monthly returns data for the S\&P 500 is available to download publicly on the internet [32].

\section{Results}

For the present study, we selected the least asymmetric (LA) wavelet (known as the Symmlet) which exhibits near symmetry about the filter midpoint. LA filters are available in even widths and the optimal filter width is dependent on the characteristics of the signal and the length of the data. The filter width chosen for this study was the LA8 (where the 8 refers to the width of the scaling function) since it enabled us to accurately calculate wavelet Betas and Correlations to the $4^{\text {th }}$ scale given the length of data available. Although the MODWT can accommodate any level $J_{0}$, in practise the largest level is chosen so as to prevent decomposition at scales longer than the total length of the data series, hence the choice of the $4^{\text {th }}$ scale.

\subsection{Correlation Analysis}

Using wavelets we examined the correlation between the S\&P 500 index and the Credit Suisse/Tremont Hedge Fund composite and sub-indices for different time horizons using the MODWT as described in Section 3. The correlations were calculated for scales 1,2,3 and 4 corresponding to a 2-4 month period, a 4-8 month period, a 8-16 month period and a 16-32 month period respectively, as well as for the original monthly returns data. The results are shown in Table 1.

The correlations for the Hedge Fund composite index with the S\&P vary according to the time period looked at, in a similar fashion to those of the Long/Short Equity strategy. This may be at least partly accounted for by the fact that Long/Short Equity is the component with the largest weighting in the index $(28.8 \%,[29])$. The correlation for Convertible Arbitrage, Fixed Income Arbitrage and Multi-Strategy with the S\&P is found to increase as the time horizon increases which may be evidence that these strategies hold less liquid or hard-to-price securities (resulting in a "smoothing" of their returns). The 
Table 1

\begin{tabular}{c||c|c|c|c|c} 
Scale & Original Data & 1 & 2 & 3 & 4 \\
\hline \hline Hedge Fund Index & 0.47 & 0.50 & 0.40 & 0.50 & 0.36 \\
\hline Convertible Arbitrage & 0.13 & 0.07 & 0.12 & 0.35 & 0.31 \\
Dedicated Short Bias & -0.75 & -0.78 & -0.76 & -0.80 & -0.88 \\
Emerging Markets & 0.48 & 0.59 & 0.53 & 0.47 & 0.39 \\
Equity Market Neutral & 0.37 & 0.43 & 0.41 & 0.30 & 0.13 \\
Event Driven & 0.55 & 0.56 & 0.58 & 0.71 & 0.51 \\
Fixed Income Arbitrage & 0.00 & 0.05 & -0.19 & 0.08 & 0.23 \\
Global Macro & 0.22 & 0.31 & 0.16 & 0.16 & 0.00 \\
Long/Short Equity & 0.58 & 0.59 & 0.54 & 0.60 & 0.53 \\
Managed Futures & -0.14 & 0.00 & -0.25 & -0.19 & -0.61 \\
Multi-Strategy & 0.10 & 0.03 & 0.03 & 0.16 & 0.25
\end{tabular}

Correlations between the S\&P 500 and the Credit Suisse/Tremont Hedge Fund indices

correlations for Dedicated Short Bias, Equity Market Neutral and Managed Futures are found to decrease significantly as the time horizon increases. Hence, these strategies may be good diversifiers over a longer time period and would be a useful addition to a market portfolio.

Using the Credit Suisse/Tremont Hedge Fund Composite index, we also measured the correlation between this and each of the sub-indices over different time horizons. Again, the correlations were calculated for scales 1,2,3 and 4 as above. The results are shown in Table 2 .

Table 2

\begin{tabular}{c||c|c|c|c|c} 
Scale & Original Data & 1 & 2 & 3 & 4 \\
\hline \hline Convertible Arbitrage & 0.40 & 0.23 & 0.36 & 0.53 & 0.63 \\
Dedicated Short Bias & -0.48 & -0.50 & -0.48 & -0.50 & -0.39 \\
Emerging Markets & 0.66 & 0.71 & 0.65 & 0.66 & 0.64 \\
Equity Market Neutral & 0.32 & 0.36 & 0.34 & 0.12 & 0.07 \\
Event Driven & 0.68 & 0.61 & 0.70 & 0.72 & 0.69 \\
Fixed Income Arbitrage & 0.41 & 0.28 & 0.33 & 0.57 & 0.57 \\
Global Macro & 0.85 & 0.88 & 0.87 & 0.85 & 0.79 \\
Long/Short Equity & 0.79 & 0.76 & 0.83 & 0.85 & 0.82 \\
Managed Futures & 0.17 & 0.35 & 0.22 & -0.01 & -0.58 \\
Multi-Strategy & 0.22 & 0.01 & 0.31 & 0.30 & 0.50
\end{tabular}

Correlations between the Credit Suisse/Tremont Hedge Fund Composite index and the sub-indices

The results for the inter-strategy correlations with the Hedge Fund Composite Index were somewhat similar to those with the S\&P 500. The correlation of Convertible Arbitrage, Fixed Income Arbitrage and Multi-Strategy with the Hedge Fund composite index was found to increase as the time period increased. Hence, these strategies may not offer 
such significant diversification benefits in a portfolio of hedge funds over a longer period. The correlation of Dedicated Short Bias, Managed Futures, Equity Market Neutral and Global Macro to the Composite Index decreased significantly as the time horizon increased. Hence these strategies may offer real diversification benefits over a longer time horizon. We examine the effects of the change in correlations at different time horizons on the efficient frontier of a portfolio made up of the Hedge Fund strategies in section 5.3.

\subsection{Systematic Risk Analysis}

We measured the Beta or market risk of various hedge fund strategies to the S\&P 500 over different time horizons using the MODWT. Once again, the correlations were calculated for scales 1,2,3 and 4 as defined in Section 5.1. The results are displayed in Table 3 .

Table 3

\begin{tabular}{c||c|c|c|c|c} 
Scale & Original Data & 1 & 2 & 3 & 4 \\
\hline \hline Hedge Fund Index & 0.25 & 0.25 & 0.21 & 0.31 & 0.26 \\
\hline Convertible Arbitrage & 0.04 & 0.01 & 0.03 & 0.16 & 0.19 \\
Dedicated Short Bias & -0.90 & -0.85 & -0.94 & -1.22 & -1.49 \\
Emerging Markets & 0.53 & 0.52 & 0.59 & 0.77 & 0.63 \\
Equity Market Neutral & 0.07 & 0.07 & 0.08 & 0.08 & 0.04 \\
Event Driven & 0.21 & 0.17 & 0.22 & 0.38 & 0.39 \\
Fixed Income Arbitrage & 0.00 & 0.01 & -0.05 & 0.02 & 0.08 \\
Global Macro & 0.16 & 0.23 & 0.11 & 0.12 & -0.01 \\
Long/Short Equity & 0.41 & 0.37 & 0.39 & 0.55 & 0.46 \\
Managed Futures & -0.11 & 0.00 & -0.23 & -0.22 & -0.61 \\
Multi-Strategy & 0.03 & 0.01 & 0.01 & 0.05 & 0.10
\end{tabular}

Betas of the Credit Suisse/Tremont Hedge Fund indices

The Beta of the Composite Hedge Fund Index appears to be reasonably static but the market risk of its components varies according to strategy. Convertible Arbitrage has a Beta of 0.04 using monthly data but using a $16-32$ month time horizon it has Beta of 0.19. This difference may be due to the fact that there is no exchange for Convertible Bonds and they are traded "over the counter" and hence can be illiquid and difficult to price.

The Beta of the Emerging Markets strategy increases from 0.53 to a maximum of 0.77 over an 8-16 month period. This may be due to liquidity constraints in emerging markets with light markets in equities causing instruments to be marked off non-synchronous prices, since the final traded price for an asset may have been before month end. The effect of liquidity issues in Emerging Markets has been studied in more detail in [33]. Another possible factor might be the difficulty in short-selling instruments in many emerging markets, hence these funds often employ a more traditional long-only type strategy.

The market risk of the Event Driven strategy is shown in Table 3 to increase from 0.21 to 0.39 over a longer time horizon, as is the case for Equity Long/Short strategy which 
increases from 0.41 to a maximum of 0.55 . In both cases these increases could be due to a number of factors, such as liquidity, the holding period of the manager or the time for the positions in the portfolios to reach a "fair value". Another significant consideration might be the use of options which have a non-linear returns profile and hence might lead to an increase in Beta. Furthermore Long/Short Equity managers may also hold significant positions in small capitalization stocks or illiquid private securities.

Equity Market Neutral, Fixed Income Arbitrage and Multi-Strategy all appear to have very small and static levels of market risk over all time horizons. Equity Market Neutral, as the name suggests, seeks to exploit pricing efficiencies between equity securities while simultaneously neutralising exposure to market risk. Fixed Income Arbitrage managers invest solely in Bonds (whether sovereign or corporate) and hence have little or no equity market risk. Multi-Strategy funds implement a dynamic strategy allocation to various Hedge Fund strategies. All of these strategies should provide good diversification to a portfolio made up of S\&P equities.

The market risk of the Dedicated Short Bias and Managed Futures strategies is found to decrease by a considerable amount as the time horizon increases. Dedicated ShortBias funds are, in a sense, mirrors of traditional long-only managers. The increase in the absolute value of their Beta may be due to excessive leverage in Bear Markets ${ }^{4}$ or the use of Put Options ${ }^{5}$ with a non-linear return profile. Managed Futures managers tend to use forecasting models that depend heavily on past price movements. The time horizons of their trades can vary greatly depending on the time horizon examined in their models and hence this may have a large impact on the aggregate amount of market risk held at different time intervals.

In summary, the level of market risk held by different strategies varies greatly according to strategy and the time horizon studied. Given the fact that many funds have minimum lock-up periods, during which investors cannot liquidate their capital, it is more appropriate to measure the market risk over longer time intervals. This has obvious consequences for the allocation strategies of Fund of Funds ${ }^{6}$ and Institutional investors alike.

\subsection{Efficient Frontier}

To enable us to demonstrate graphically the effects of calculating the correlation matrix of Hedge Funds using different time horizons, we show (Figure 5.3) the efficient frontier for each time horizon studied. In the calculation of the efficient frontier we have imposed constraints on the weights allowed, to prevent short-selling of Hedge Funds. This constraint is natural in the context of Hedge Funds due to the difficulties in shortselling of funds; (note that short-selling may be achievable by the use of swaps but is uncommon) [30,31]. Each of these efficient frontiers is calculated using the annualised returns and variance from the original data. Hence, the results we see in Figure 5.3 are purely dependent on the correlation matrix calculated at each time horizon. The efficient

$\overline{4 \text { A Bear }}$ Market is considered to be a prolonged period in which asset prices fall, accompanied by widespread pessimism

5 A put is an option contract that gives the holder the right to sell a certain quantity of an underlying security to the writer of the option, at a specified price up to a specified date

6 A Hedge Fund which invests in other hedge funds 


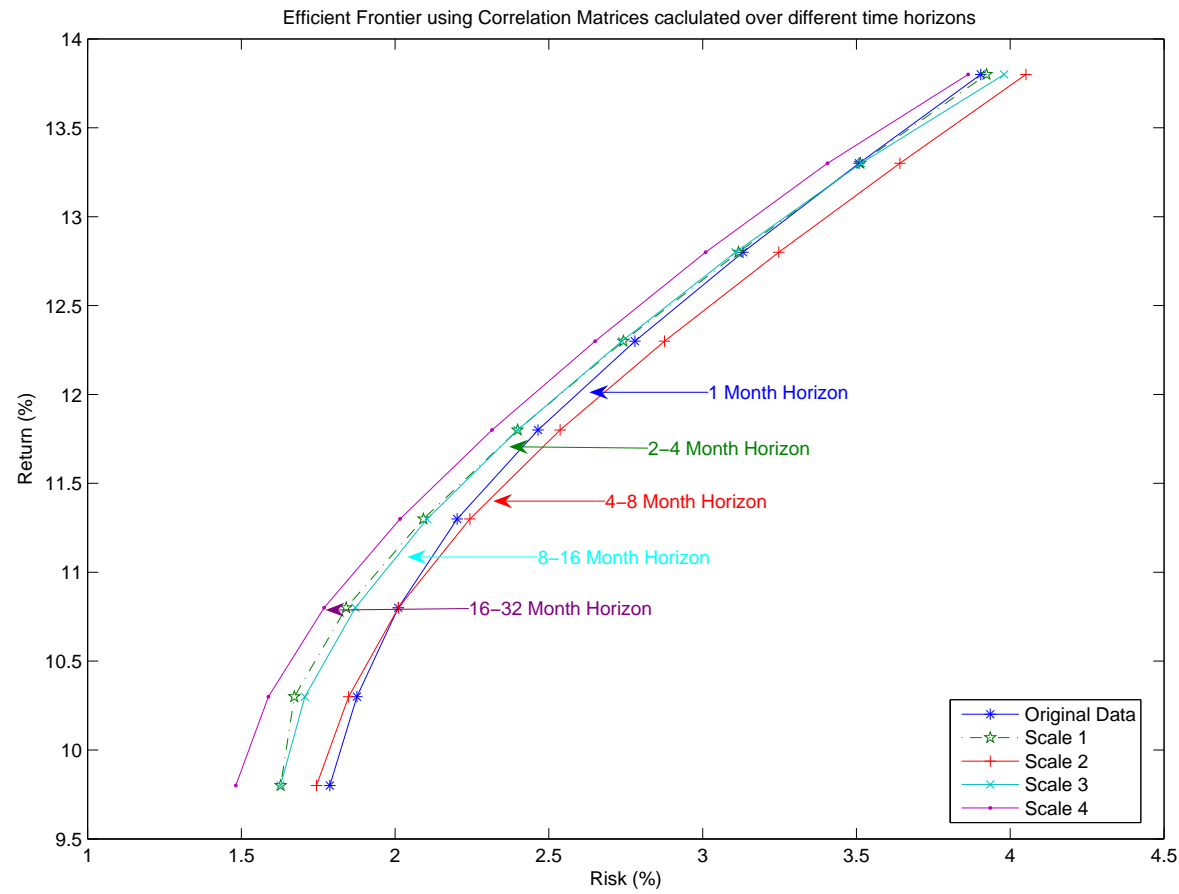

Fig. 1. Efficient Frontier using Correlation Matrices calculated over different time scales

frontiers are calculated using the techniques described in Section 2.1 and consist of just the Credit Suisse/Tremont sub-indices.

At lower return values the level of risk calculated for the original data is higher than that calculated at all other scales. As the return level increases, the risk of the portfolio (created using the correlation matrix calculated using the monthly returns), decreases relative to the others. However, the lowest risk portfolio overall is found using a correlation matrix calculated over a 16-32 month horizon (Scale 4). These findings have significant implications for investors allocating capital to Hedge Funds.

\section{Conclusions}

The MODWT was applied to demonstrate the dependence of the correlation and market risk of Hedge Funds on the coarse/fine graining of Hedge Fund time series. This dependence was found to apply for correlations between Hedge Fund sub-strategies and both the S\&P 500 and the Hedge Fund composite index. In particular, the correlation between Convertible Arbitrage, Fixed Income Arbitrage and Multi-Strategy and both the S\&P and Hedge Fund Composite index was found to increase as the time scale increased. The correlation between Dedicated Short Bias, Equity Market Neutral, Global Macro and Managed Futures strategies with the S\&P and the Hedge Fund Composite 
index was found to decrease as the time scale increases. This decrease in correlation over longer time scales for certain strategies is in contrast to the Epps effect $[16,17]$ where the correlation between financial time series was shown to increase over long time scales. However, in the case of the Epps effect the time scales studied were much shorter [17] $\left(0-2 \frac{1}{2}\right.$ hours $)$.

The level of market risk held by different Hedge Fund strategies was also found to vary according to the time horizon studied. The level of market risk of Convertible Arbitrage, Emerging Markets, Event-Driven and Long/Short Equity was found to increase as the time scale increased. The market risk of Dedicated Short Bias, Global Macro and Managed Futures was found to decrease as the time scale increased.

The minimum lock-up times applicable to many Hedge Funds mean that it may be more suitable to use correlation matrices calculated using longer time scales. To demonstrate this, we calculated the efficient frontier for four different time scales, as well as for the original data for a portfolio constructed from the sub-strategies. It was found that the level of risk of a portfolio of funds varies according to time-scale with the lowest risk portfolio attained using a scale of $16-32$ months. This scale is significant as Hedge Funds often have lock-up period of $12-24$ months inside of which investors cannot withdraw their invested capital [30].

Interesting future work worth considering include the measurement of the Alpha (Equation 4) of different strategies over different time scales. In the case of Hedge Funds the Alpha is a measure of the abnormal return, which is the value added by the manager. A Hedge Fund manager who appears to add Alpha, using monthly returns, may actually be found to just hold market risk over longer time scales and hence be less interesting to investors. Additional insights could be expected through the analysis of the levels of correlation and market risk of Hedge Funds with, for example, a bond index, a world equity index and a small-cap equity index over different scales using a multi-factor regression model.

\section{References}

[1] The Barclay Group, www.barclayhedge.com, accessed on 4th April 2008.

[2] Schneeweis, T., Martin, G., Journal of Alternative Investments 4, (2001) 7-26.

[3] Liang, B., Financ. Anal. J. 57, (2001) 1.

[4] Nishiyama, N., Physica A 301 (1-4) (2001) 457-472.

[5] Miceli, M.A., Susinno, G., Physica A 344 (1-2) (2004) 95-99.

[6] Conlon, T., Ruskin, H.J., Crane, M., Physica A 382 (2) (2007) 565-576.

[7] Perello, J., Physica A 383 (2) (2007) 480-496.

[8] Whitcher, B.J., Guthorp, P., Percival, D.B., J. Geophys. Res., 105(D11), (2000) 14941-14962.

[9] Cornish, C.R., Bretherton, C.S., Percival, D.B., Bound-Lay. Meteorol. 119 (2006) 339-374.

[10] Benzi. R., Biferale, L., Toschi, F., Phys. Rev. Lett. 80 (1998) 3244 - 3247.

[11] Ramsey, J.B., Lampart, C., Macroecon. Dyn., 2(1), 1998, 49-71

[12] Gencay, R., Selcuk F., Whitcher B., Physica A 289, (2001) 249-266.

[13] Gencay, R., Selcuk F., Whitcher B., Physica A 289, (2001) 543-556.

[14] Gencay, R., Selcuk F., Whitcher B., Quant. Financ. 3, (2003) 108-116.

[15] Gencay, R., Selcuk F., Whitcher B., J. Int. Money Finance 24, (2005) 55-77.

[16] Epps, T.W., J. Am. Stat. Assoc 74, (1979) 291-298.

[17] Tóth, B., Kertész, J., Physica A 383 (2007) 54-84.

[18] Sharpe, W.F., J. Finance 35, 910-919.

[19] Markowitz, H., Portfolio Selection: Efficient diversification of investments (John Wiley \& sons, 1959). 
[20] Elton, E.J., Gruber, M.J., Modern Portfolio Theory and Investment Analysis, 6th Edition (John Wiley \& Sons, 2002).

[21] Bouchaud, J. P., Potters. M, Theory of Financial Risk and Derivative Pricing (Cambridge University Press, 2003).

[22] Gencay, R., Selcuk, B., Whitcher, B., An Introduction to Wavelets and other Filtering Methods in Finance and Economics (Academic Press, 2001).

[23] Sharkasi, A., Ruskin, H.J., Crane, M., Int. J. Theoretical Appl. Finance 8(5), (2005) 603-622.

[24] Sharkasi, A., Crane, M., Ruskin, H.J., Matos, J.A., Physica A 368 (2) (2006) 511-521.

[25] Daubechies, I., Ten lectures on Wavelets (SIAM, 1992).

[26] Bruce, A., Gao, H., Applied Wavelet Analysis with S-Plus (Springer-Verlag, 1996).

[27] Burrus, C.S., Gopinath, R.A., Gao, H., Introduction to wavelets and wavelets transforms (Prentice Hall 1997).

[28] Percival, D.B., Walden, A.T., Wavelet methods for time series analysis (Cambridge University press, 2000).

[29] Credit Suisse/Tremont Hedge Fund Indices, www.hedgeindex.com, accessed on 11th January 2008.

[30] L'habitant, F.S., Hedge Funds (Wiley, 2002).

[31] L'habitant, F.S., Hedge Funds - Quantitative Insights (Wiley, 2004).

[32] S\&P 500 Index returns data, finance.yahoo.com, accessed on 11th January 2008.

[33] Wilcox, D., Gebbie, T., Physica A 344 (1-2) (2004) 294-298. 